\title{
One-dimensional shock-capturing for high-order discontinuous Galerkin methods
}

\author{
E. Casoni ${ }^{1}$, J. Peraire ${ }^{2}$ and A. Huerta ${ }^{1, *, \dagger}$ \\ ${ }^{1}$ Laboratori de Calcul Numeric (LaCaN). Departament de Matematica Aplicada III E.T.S. de Ingenieros de Caminos, \\ Canales y Puertos, Universitat Politecnica de Catalunya, Jordi Girona 1-3, 08034 Barcelona, Spain \\ ${ }^{2}$ Department of Aeronautics and Astronautics, Massachusetts Institute of Technology, Cambridge, MA 02139, USA
}

\begin{abstract}
SUMMARY
Discontinuous Galerkin methods have emerged in recent years as an alternative for nonlinear conservation equations. In particular, their inherent structure (a numerical flux based on a suitable approximate Riemann solver introduces some stabilization) suggests that they are specially adapted to capture shocks. However, numerical fluxes are not sufficient to stabilize the solution in the presence of shocks. Thus, slope limiter methods, which are extensions of finite volume methods, have been proposed. These techniques require, in practice, mesh adaption to localize the shock structure. This is is more obvious for large elements typical of high-order approximations. Here, a new approach based on the introduction of artificial diffusion into the original equations is presented. The order is not systematically decreased to one in the presence of the shock, large high-order elements can be used, and several linear and nonlinear tests demonstrate the efficiency of the proposed methodology.
\end{abstract}

KEY WORDS: discontinuous Galerkin; shock capturing; artificial viscosity; high-order approximation

\section{INTRODUCTION}

In the last decades, discontinuous Galerkin (DG) methods have centered many studies for nonlinear conservation laws and convection-dominated problems. Some CFD applications may require highly accurate numerical models to obtain high fidelity predictions. Solutions of nonlinear PDEs may develop discontinuities in finite time even if the initial condition is smooth. The inherent stability of DG methods produces adequate solutions but only for constant or linear approximations and fine enough discretizations. Note, however, that standard high-order DG approximations require further stabilization.

Limiting techniques have emerged as a solution for this problem. See, for instance, Runge-Kutta discontinuous Galerkin (RKDG) methods $[1,2]$. They are a special class of explicit RK schemes [3-5] that combine with approximate Riemann solvers and nonlinear operators (i.e., slope limiters) to satisfy stability. Limiters were initially designed in the context of finite volumes [6].

Classical adaptive procedures [1,7], which are based on limiting techniques, drastically reduce the order of the approximations in the vicinity of shocks. This induces a method called total variation bounded in the means, which at most is second order in space. Moreover, explicit RKDG methods are at most fourth order in time. Note also that, to the authors' knowledge, no implicit time integration schemes have been developed in combination with slope limiters. In summary, standard

\footnotetext{
*Correspondence to: A. Huerta, Laboratori de Càlcul Numèric (LaCàN), E.T.S. Ingenieros de Caminos, Universitat Politècnica de Catalunya, Jordi Girona 1, E-08034 Barcelona, Spain.

${ }^{\dagger}$ E-mail: antonio.huerta@upc.edu
} 
limiting techniques add numerical diffusion of the order of the mesh size. This implies that mesh adaption (refinement) procedures are needed. Recall that adaption should incorporate mesh directionality because shocks are lower dimensional structures. To overcome these drawbacks, methods limiting high-order moments have been proposed [8,9]. However, they also require refined meshes.

Another alternative is high-order non-oscillatory reconstruction. Researchers' attention are focused on essentially non-oscillatory and weighted essentially non-oscillatory techniques, see for instance $[4,10]$, because they preserve nonlinear stability and retain the order of the interpolation (using additional degrees of freedom [DOF] to resolve sharp profiles). However, their computational overhead is excessive for high-order approximations, they require structured grids, and they may loose robustness for high-order schemes. Other alternatives exploiting the DG inherent numerical flux stabilization are possible [11].

Here, an obvious and, in principle, simple approach, already introduced in the 1950s [12], is advocated: adding dissipation to obtain stable solutions. In fact, reincorporating this relevant physics [13] in nonlinear hyperbolic conservation is not trivial. That is, the inherent difficulties in determining where and how much viscosity must be introduced to avoid oscillations without causing unnecessary smearing have deterred the extensive use of this approach. Recently, in [14,15], an artificial viscosity term (based on the mesh size $h$ and the degree of the interpolating polynomial $p$ ) has been proposed in conjunction with a discontinuity detection procedure.

The introduction of dissipation in the equation does not hinder the use of high-order and implicit schemes, in contrast with RKDG methods. Introducing an artificial viscosity that scales with the DG resolution length scales, $h / p$, makes the shock width also scale in the same manner. Here, following [16], the extensive experience in limiting techniques is used to define an artificial diffusion method. The obtained value for the viscosity scales such as $\varepsilon \sim \mathcal{O}\left(h^{k}\right)$ for some $1 \leqslant k \leqslant p$. Numerical tests show consistently that $h^{k}$ is smaller than $h / p$, proposed in [14]. Moreover, sharp shock profiles free of spurious oscillations are obtained. The order of the approximation is also maintained, and the method allows to use coarse meshes. The artificial diffusion method is performed in combination with the shock sensor proposed in [14] to further ensure that no artificial diffusion is added in smooth regimes.

Numerical examples suggest that constant element-by-element artificial diffusion is, in general, sufficient. Nevertheless, an extension to piecewise constant diffusion inside each element is also proposed. This subcell approach, although requiring further research, seems promising in the presence of large element with sharp shocks. In fact, subcell detection and viscosity show more accurate solutions.

The introduction of the artificial viscosity term requires the discretization of second-order derivatives with DG methods. Several DG methods have been proposed for elliptic operators [17-21]. Here, for simplicity, the local discontinuous Galerkin (LDG) approach of Cockburn and Shu [22] is used, but other methods can also be employed, see for instance [21].

This paper is structured as follows: the LDG method is briefly presented in Section 2, mostly for notation purposes. The necessary background related to slope limiting techniques is reviewed in Sections 3.1 and 3.2. In Section 3.3, the proposed artificial diffusion technique is presented in detail, and the discontinuity sensor is described in Section 3.4. Section 4 presents an extension to piecewise constant subcell viscosity. Numerical tests demonstrate the efficiency of the method in Section 5.

\section{THE BASICS OF LDG IN ONE DIMENSION}

Consider a scalar conservation law

$$
\left.u_{t}+f_{x}(u)=0, \quad x \in\right] 0,1[, t>0
$$

and introduce a dissipative term, the artificial viscosity, of the form

$$
\left.u_{t}+f_{x}(u)-\left(\varepsilon(u) u_{x}\right)_{x}=0, \quad x \in\right] 0,1[, t>0 .
$$

Here, $\varepsilon(u)$ is a non-negative quantity that models the artificial diffusion as a nonlinear coefficient depending on the solution $u$. Notice that adding viscosity to the original equations introduces 
second-order derivatives, which require special attention in discontinuous approximations. Several methods have been proposed to deal with this situation; here, LDG has been used, see the seminal paper [22].

To apply the LDG method, Equation (2) is written as a system of first-order hyperbolic equations, typical of mixed methods

$$
\begin{aligned}
& \left.u_{t}+f_{x}(u)-\sigma_{x}=0, \quad x \in\right] 0,1[, t>0 \\
& \left.\sigma-\varepsilon(u) u_{x}=0, \quad x \in\right] 0,1[.
\end{aligned}
$$

where the auxiliary variable $\sigma$ has been introduced. To simplify the developments and following [22], the new variable $g(u)=\int^{u} \varepsilon(s) d s$ is introduced, directly relate to $\sigma$; note that by using Leibniz rule, it is easily demonstrated that $\sigma=g_{x}(u)$.

Let $\left\{I_{e}\right\}_{e=1, \ldots, J}$ with $I_{e}=\left[x_{e}, x_{e+1}\right]$ be a partition of the interval $[0,1]$ into $J$ subintervals, with $0=x_{1}<x_{2}<\cdots<x_{J+1}=1$. Because of the discontinuity of the test functions, the integral form of Equation (3) in DG is stated element by element. Consider test functions $v$ and $\tau$ in $\mathcal{P}^{p}\left(I_{e}\right)$, with $\mathcal{P}^{p}\left(I_{e}\right)$ being the space of polynomial functions of total degree $\leqslant p$ defined over $I_{e}$. An approximation to the exact solution of (3), also denoted by $u$ and $\sigma$ in an abuse of notation, is obtained if

$$
\begin{aligned}
\int_{I_{e}} u_{t} v d x-\int_{I_{e}}(f(u)-\sigma) v_{x} d x+\left[\hat{f}_{e+1} v\left(x_{e+1}^{-}\right)-\hat{f}_{e} v\left(x_{e}^{+}\right)\right]- \\
{\left[\hat{\sigma}_{e+1} v\left(x_{e+1}^{-}\right)-\hat{\sigma}_{e} v\left(x_{e}^{+}\right)\right]=0 } \\
\int_{I_{e}} \sigma \tau d x+\int_{I_{e}} g(u) \tau_{x} d x-\left[\hat{g}_{e+1} \tau\left(x_{e+1}^{-}\right)-\hat{g}_{e} \tau\left(x_{e}^{+}\right)\right]=0
\end{aligned}
$$

for all $v$ and $\tau$ in $\mathcal{P}^{p}\left(I_{e}\right)$ and for every element $e=1, \ldots, J$ and $t>0$.

The nonlinear flux $f(u)$ has been replaced by numerical one $\hat{f}=\hat{f}(u)$, which is typically chosen as the Roe or Lax-Friedrichs flux. For implementation details, see [20]. There are two other fluxes due to LDG methods, $\hat{\sigma}$ and $\hat{g}$, which can be seen as approximations of the numerical traces of $\sigma$ and $g(u)$ on the boundaries. Standard numerical fluxes are used here, see [22]. Note that at interfaces, the following notation is used:

$$
x_{e}^{ \pm}=\lim _{\epsilon \longrightarrow 0} x_{e} \pm \epsilon .
$$

It is usual to assume the $\varepsilon(u)$ constant element by element, see [14]. Here, the same assumption is used, and thus, for element $e, g(u)=\varepsilon_{e} u$, that is, Equation (4b) becomes

$$
\int_{I_{e}} \sigma \tau d x+\int_{I_{e}} \varepsilon_{e} u \tau_{x} d x-\varepsilon_{e}\left[\hat{u}_{e+1} \tau\left(x_{e+1}^{-}\right)-\hat{u}_{e} \tau\left(x_{e}^{+}\right)\right]=0 .
$$

\section{Remark 1}

Other assumptions for the distribution of $\varepsilon(u)$ are possible. In particular, assuming continuous $\varepsilon(u)$ precludes incoherences in (2) because of a discontinuous diffusion. This analysis, however, is beyond the scope of the present paper.

\section{PROPOSED APPROACH FOR THE ARTIFICIAL DIFFUSION}

The computation of the amount of artificial viscosity is performed by combining the ideas of slope limiters and shock-capturing methods. The popular RKDG method [20] and the generalization of the classical slope limiter [1], $\Lambda \Pi_{h}$, proposed in [8] provide a frame of reference for the present work. The objective of this section is, first, to briefly describe the construction of RKDG method and, second, to explain in detail how to compute the amount of artificial diffusion to introduce in (2). 


\subsection{RKDG methods}

RKDG methods provide stable and high-order accurate schemes for nonlinear convectiondominated problems. The DG space discretization, the special strong stability preserving RK time discretizations, and the generalized slope limiters $\Lambda \Pi_{h}$ are intertwined just in the right way to achieve nonlinear stability. Note that $h$ is a characteristic measure of the elements.

Consider the conservation law (1) and approximate $-f_{x}(u)$ by a DG space discretization. A system of ODEs of the form $u_{t}=L(u)$ is obtained. The total variation diminish RK (TVD RK) time discretization scheme introduced in [3] is used to integrate the ODE system in time. Let $\left\{t^{n}\right\}_{n=0, \ldots, N}$ be a partition of $[0, T]$. The general form for an $m$-stage scheme reads as:

- Set $u^{0}=\Lambda \Pi_{h}\left(u_{0}\right)$, where $u_{0}$ is the initial data for (1).

- For $n=0, \ldots, N-1$, compute $u^{n+1}$ from $u^{n}$ as:

- Set $u^{(0)}=u^{n}$

- For $\ell=1, \ldots, m$, compute the intermediate functions:

$$
u^{(\ell)}=\Lambda \Pi_{h}\left(u^{n+k_{\ell}}\right), \quad u^{n+k_{\ell}}=\sum_{i=0}^{\ell-1} \alpha_{\ell i} w^{\ell i}, \quad w^{\ell i}=u^{(i)}+\frac{\beta_{\ell i}}{\alpha_{\ell i}} L\left(u^{(i)}\right)
$$

- Set $u^{n+1}=u^{(m)}$

where the coefficients $\alpha_{\ell i}$ and $\beta_{\ell i}$ must satisfy typical RK conditions, see [20]. This is the general form of the RKDG methods. They are stable under a CFL condition, see [20], in the seminorm $|\cdot|_{\mathrm{TV}}$. That is, $\left|\bar{u}^{n+1}\right|_{\mathrm{TV}} \leqslant\left|\bar{u}^{n}\right|_{\mathrm{TV}}$ where $|\bar{u}|_{\mathrm{TV}}=\sum_{e}\left|\bar{u}_{e+1}-\bar{u}_{e}\right|$, with $\bar{u}$ being the average of $u$ in the element.

Notice that limiting techniques are implemented in two steps for each stage of the RKDG scheme. First, the conservation law (1) is solved, and second, the limiting operator $\Lambda \Pi_{h}$ is applied.

Assuming time integration under enough regularity, the weak formulation for the $k_{\ell}$-stage can also be symbolically written, for all $v \in \mathcal{P}^{p}\left(I_{e}\right), e=1, \ldots, J$ and $t>0$, as

$$
\int_{I_{e}} u^{n+k_{\ell}} v d x=\int_{I_{e}} u^{n} v d x+\int_{t^{n}}^{t^{n+k_{\ell}}}\left\{\int_{I_{e}} f(u) v_{x} d x-\left[\hat{f}_{e+1} v\left(x_{e+1}^{-}\right)-\hat{f}_{e} v\left(x_{e}^{+}\right)\right]\right\} d t,
$$

and the limiting step is then applied

$$
u^{(\ell)}=\Lambda \Pi_{h}\left(u^{n+k_{\ell}}\right)
$$

\subsection{The nonlinear limiting operator}

Slope limiting techniques rely on the construction of a nonlinear local projection operator $\Lambda \Pi_{h}$ whose aim is to enforce nonlinear stability of the approximate solution. For simplicity, the approximate solution is expanded in terms of orthogonal Legendre polynomial; within each element $I_{e}$ (for all $e=1, \ldots, J)$, the approximation of $u$ may be written as

$$
u^{e}(x, t)=\sum_{i=0}^{p} c_{i}^{e}(t) P_{i}(x)
$$

where $P_{i}$ are the Legendre polynomials normalized such that $P_{i}(1)=1$, and the DOF $c_{i}^{e}$ are the so-called modal coefficients.

Biswas et al. [8] define a high-order slope limiter relying on the Total Varitation Diminishing in the means (TVDM) version of the generalized one proposed in [1], which is designed for linear approximations, that is, $p=1$. The idea is to limit the solution by limiting its coefficients. Beginning with the coefficient associated with the highest polynomial degree (i.e., for $i=p, p-1, \ldots, 1$ ), the coefficient $c_{i}^{e}$ is replaced by

$$
\tilde{c}_{i}^{e}=\operatorname{minmod}\left(c_{i}^{e},\left(c_{i-1}^{e+1}-c_{i-1}^{e}\right) /(2 i-1),\left(c_{i-1}^{e}-c_{i-1}^{e-1}\right) /(2 i-1)\right),
$$


with the usual definition:

$$
\operatorname{minmod}\left(a_{1}, a_{2}, a_{3}\right)= \begin{cases}s \min _{1 \leqslant n \leqslant 3}\left|a_{n}\right| & \text { if } s=\operatorname{sign}\left(a_{1}\right)=\operatorname{sign}\left(a_{2}\right)=\operatorname{sign}\left(a_{3}\right), \\ 0 & \text { otherwise. }\end{cases}
$$

The limiter is active when $\tilde{c}_{i}^{e} \neq c_{i}^{e}$ for any $i$. Thus, the reconstructed coefficient $\tilde{c}_{i}^{e}$ limits $c_{i}^{e}$, which, in the absence of discontinuities, is proportional to the $i$ th derivative of the approximation. Obviously, to preclude spurious oscillations, higher order derivatives are limited first. The reconstructed solution has typically the following structure

$$
\Lambda \Pi_{h}\left(u^{e}\right)(x, t)=\sum_{i=0}^{k} c_{i}^{e}(t) P_{i}(x)+\sum_{i=k+1}^{p} \tilde{c}_{i}^{e}(t) P_{i}(x) .
$$

A major improvement of this reconstruction is that rarely $k$ is as low as 0 , which is the default value for the limiter proposed in [1].

In the next section, the proposed artificial viscosity approach is presented. Note that the superscript ${ }^{e}$ indicating the element number is dropped to simplify the presentation. The nonlinear convection-diffusion equation, see (2), is solved instead of applying slope limiters to the numerical solution of the nonlinear hyperbolic equation, see (1). The heuristics behind the construction of approximate Riemann solvers and slope limiters described in [13] are the guiding lines to determine an explicit expression for the artificial diffusion term. A relation between the artificial viscosity $\varepsilon(u)$ in (2) and the limited solution, see (7), of (1) is established.

\subsection{Artificial diffusion}

Denote by $u^{\varepsilon}$ the exact solution of (2). The weak form of this convection-diffusion equation is

$$
\int_{I_{e}} u_{t}^{\varepsilon} v d x-\int_{I_{e}}\left(f\left(u^{\varepsilon}\right)-\varepsilon\left(u^{\varepsilon}\right) u_{x}^{\varepsilon}\right) v_{x} d x+\left[\left(f\left(u^{\varepsilon}\right)-\varepsilon\left(u^{\varepsilon}\right) u_{x}^{\varepsilon}\right) v\right]_{x_{e}}^{x_{e+1}}=0
$$

where the same partition proposed in Section 2 is used.

It is well known, see for instance $[6,13]$, that for physical reasons, the correct solution, called the entropy solution, is obtained when viscosity tends to zero, that is, $\varepsilon \rightarrow 0$. Therefore, the entropy solution at a given instant $t$ is defined by

$$
\int_{I_{e}} u v d x=\lim _{\varepsilon \rightarrow 0} \int_{I_{e}} u^{\varepsilon} v d x
$$

and analogously, the numerical flux, typical of DG methods, is

$$
\hat{f}_{e}=\lim _{\varepsilon \rightarrow 0}\left[f\left(u^{\varepsilon}\left(x_{e}, t\right)\right)-\varepsilon\left(u^{\varepsilon}\right) u_{x}^{\varepsilon}\left(x_{e}, t\right)\right] .
$$

Taking the limit as $\varepsilon$ goes to zero in (8) and by following the procedures developed in [13], the following weak formulation is obtained:

$$
\begin{aligned}
\int_{I_{e}} u_{t} v d x-\int_{I_{e}} f(u) v_{x} d x+\left[\hat{f}_{e+1} v\left(x_{e+1}^{-}\right)-\hat{f}_{e} v\left(x_{e}^{+}\right)\right] & \\
& +\int_{I_{e}} \hat{\varepsilon}(u) u_{x} v_{x} d x=0
\end{aligned}
$$

where the last term in (8) has been replaced by $\left[\hat{f}_{e+1} v\left(x_{e+1}^{-}\right)-\hat{f}_{e} v\left(x_{e}^{+}\right)\right]$. The term containing the artificial viscosity coefficient $\hat{\varepsilon}(u)$ is the so-called shock-capturing term, and it is determined in what follows.

\section{Remark 2}

The numerical flux $\hat{f}$ given by (9) can be reduced to an E-flux of the form $\hat{f}_{e}=f\left(u\left(x_{e}, t\right)\right)$ such as the Godunov flux, the Enquist-Osher flux, or the Lax-Friedrichs flux for $\Delta t$ small enough. For a justification, see [23]. 
Assuming again time integration under enough regularity, the weak formulation is then rewritten as

$$
\begin{aligned}
\int_{I_{e}} u^{(\ell)} v d x= & \int_{I_{e}} u^{n} v d x+\int_{t^{n}}^{t^{n+k_{\ell}}} \int_{I_{e}} f(u) v_{x} d x d t \\
& -\int_{t^{n}}^{t^{n+k_{\ell}}}\left[\hat{f}_{e+1} v\left(x_{e+1}^{-}\right)-\hat{f}_{e} v\left(x_{e}^{+}\right)\right] d t-\int_{t^{n}}^{t^{n+k_{\ell}}} \int_{I_{e}} \hat{\varepsilon}(u) u_{x} v_{x} d x d t .
\end{aligned}
$$

To determine the shock-capturing term, the RKDG scheme described in (5) is replaced into (11) to obtain

$$
\int_{I_{e}} \Lambda \Pi_{h}\left(u^{n+k_{\ell}}\right) v d x=\int_{I_{e}} u^{n+k_{\ell}} v d x-\int_{t^{n}}^{t^{n+k_{\ell}}} \int_{I_{e}} \hat{\varepsilon}(u) u_{x} v_{x} d x d t
$$

Assuming $\hat{\varepsilon}$ constant for $x \in I_{e}$ and $t \in\left[t^{n}, t^{n+k_{\ell}}\right]$, an explicit expression for the artificial diffusion coefficient is obtained

$$
\hat{\varepsilon}=\left(\int_{I_{e}}\left(u^{n+k_{\ell}}-\Lambda \Pi_{h}\left(u^{n+k_{\ell}}\right)\right) v d x\right) /\left(\int_{t^{n}}^{t^{n+k_{\ell}}} \int_{I_{e}} u_{x} v_{x} d x d t\right)
$$

where $u^{n+k_{\ell}}$ is the standard DG solution (not limited) obtained from (5a), and $\Lambda \Pi_{h}\left(u^{n+k_{\ell}}\right)$ is the reconstructed solution (5b), whose structure is described in (7).

In fact, for each Legendre polynomial $v=P_{i}, i=1, \ldots, p$, a viscosity is obtained, say $\left\{\hat{\varepsilon}_{i}\right\}_{i=1, \ldots, p}$. Thus, each viscosity $\hat{\varepsilon}_{i}$ contains information of the reconstructed moment $\tilde{c}_{i}$. Of course, from (7) and (13), it is obvious that $\hat{\varepsilon}_{i}=0$ for $i=0, \ldots, k$.

By using the orthogonality and hierarchy of Legendre polynomials in (13), viscosities are readily computed

$$
\hat{\varepsilon}_{i}=\frac{h^{2}\left(c_{i}^{e}-\tilde{c}_{i}^{e}\right)}{2(2 i+1)}\left[\int_{t^{n}}^{t^{n+k_{\ell}}} \int_{-1}^{1} u_{\xi} \frac{d P_{i}}{d \xi} d \xi d t\right]^{-1} \quad \text { for } i=k+1, \ldots, p,
$$

where $h$ is the element size, and $\xi$ are the local coordinates in the reference element $[-1,1]$.

Only the maximum viscosity is retained because it corresponds to the lower order reconstructed moment

$$
\hat{\varepsilon}=\max \left\{\hat{\varepsilon}_{k+1}, \ldots, \hat{\varepsilon}_{p}\right\}=\hat{\varepsilon}_{\min \left\{i||_{i}^{e} \neq c_{i}^{e}\right\}}
$$

and ensures that the method is slightly on the diffusive side.

3.3.1. Order of the introduced diffusion. There are two key points to ensure the success of a shockcapturing method. First, it is crucial to preserve accuracy in smooth regions, and second, it is required to obtain sharp shock profiles, thinner than the element size.

Assuming sufficient regularity in (13) and (15), the order of the introduced artificial diffusion can be inferred by a simple analysis on the basis of the modal coefficients of the approximation.

Under this assumption, that is, in the absence of discontinuities, the DOF $c_{j}^{e}(t)$ of an approximation of the form (6) scale as $h^{j}$ for $j=0, \ldots, p$, see $[9,24]$. Moreover, by using basic orthogonality properties of Legendre polynomials and assuming $c_{j}(t)$ constant on $\left[t^{n}, t^{n+k_{l}}\right]$, a Taylor analysis of (13) demonstrates that the introduced viscosity scales like

$$
\hat{\varepsilon} \sim \begin{cases}\mathcal{O}\left(h^{k+1} / \Delta t\right) & \text { if } k \text { is even } \\ \mathcal{O}\left(h^{k+2} / \Delta t\right) & \text { if } k \text { is odd }\end{cases}
$$

where $k$ is the last nonreconstructed moment in (7). Note that the time step $\Delta t$ is chosen to satisfy the CFL condition in every step of the TVD-RKDG methods; consequently, $\Delta t \sim \mathcal{O}(h)$, see [20]. 
In contrast with more standard approaches [1] that induce a shock profile of $\mathcal{O}(h)$, the proposed diffusion scales as $\mathcal{O}\left(h^{k}\right)$ for $1 \leqslant k \leqslant p$. Numerical examples indicate that, in general, $k>1$. Thus, accuracy is higher than the one in the shock regions. Moreover, the examples also show that for relatively coarse meshes and high-order approximation, the proposed diffusion is smaller than those proposed in [14,15], which scales like $\hat{\varepsilon} \sim \mathcal{O}(h / p)$.

\subsection{Shock detection}

Introducing just the necessary diffusion is important, but it is also crucial to do it where it is needed. In principle, with the approach proposed in the previous section, it may seem that there is no need for any discontinuity sensor. Because if no limiting is necessary, the artificial diffusion is zero. Thus, in a sense, the proposed approach already incorporates a discontinuity sensor. Nevertheless, as it will become clear in the first numerical example, without a proper shock detection, the approach is overdiffusive. Two key aspects contribute to this over-diffusion: constant element-by-element artificial diffusion and the proposed algorithm that always takes the maximum computed diffusion. Using the discontinuity sensor restricts the artificial diffusion to elements where it is absolutely necessary and, moreover, reduces the computation overhead by evaluating the artificial diffusion only to the elements detected.

The smoothness indicator proposed in [14] for each element is used here because it is very efficient in the presence of high-order elements (standard in DG),

$$
S_{I_{e}}=\left(\int_{I_{e}}|u-\hat{u}|^{2} d x\right) /\left(\int_{I_{e}}|u|^{2} d x\right) .
$$

Here, $\hat{u}$ represents the approximation of order $p-1$ and $u$ the approximation of order $p$.

Assuming that the polynomial expansion behaves like the Fourier one, the sensor gives an idea of the regularity of the approximate solution. In particular, if $S_{I_{e}}>p^{-4}$, the approximation $u$ is expected to be at most $\mathcal{C}^{0}$ for $e=1, \ldots, J$. In this case, $\hat{\varepsilon}>0$ in $I_{e}$. Otherwise, the coefficients of the approximate solution decay as expected, and no artificial diffusion is needed, see [25] for details. This criterion is based on the well-known Fourier approximation properties [26]. Namely, for any function $f(x)$ expanded in terms of a periodic Fourier approximation $S F(f)=\sum_{n=-\infty}^{\infty} g_{n} e^{i n x}$, if $f(x) \in \mathcal{C}^{k-1}$, and $\partial^{k} f / \partial x^{k}$ is piecewise continuous, then $\left|g_{n}\right| \sim n^{-k}$ for $n \longrightarrow \infty$.

\section{SUBCELL EXTENSION FOR THE ARTIFICIAL DIFFUSION METHOD}

In this setting, the discontinuity sensor (16) is an element-based integral, and $\varepsilon(u)$ takes constant value within each element. However, from (2), one can think that the discrete approximation generated by the numerical scheme is an exact solution to a slightly perturbed PDE of the original conservation law (1). According to this, the ideal diffusion should be a continuous function not only depending on the solution but also point-wise defined, that is, $\varepsilon(u(x))$. However, the shock location for a given flow field is rarely known a priori, and the artificial viscosity cannot be a predetermined function in space. Moreover, a point-wise switch, based on purely local quantities, is not yet a dependable option at higher order interpolations because of the severe numerical noise in the shock layer.

Nevertheless, constant artificial viscosity within each element may have some drawbacks. Namely, it spans over one element, which can be relatively large compared with the shock width, and moreover, it introduces discontinuities in the derivatives that may lead to artificially nonsmooth solutions.To address the former issue, in elements where the shock width is far more thinner than the element size, a more local diffusion may be beneficial, especially in terms of accuracy. The piecewise discontinuous diffusion matter is not addressed here. However, the extension of the proposed artificial diffusion into a division of subcells within each element, as proposed next, reduces the diffusion jumps and, consequently, reduces slightly the influence of the diffusion jumps. 


\subsection{Subcell shock detection}

First, it is necessary to identify more precisely the shock within the element. To this purpose, the computational element $I_{e}$ is divided into small patches of size $h / p$, named subcells, and the discontinuity sensor (16) is applied over each one of them. This procedure is only performed in those elements flagged by the element-wise discontinuity sensor.

Here, for simplicity, the subcells are a uniform partition of element $I_{e}=\left[x_{k}, x_{k+1}\right]$. Equally spaced interior nodes of the high-order mesh define the subdivision. Hence, each element is divided into a set of $p$ non-overlapping intervals of uniform size, that is,

$$
I_{e}=\bigcup_{l=1}^{p} I_{e}^{l} \text { with } I_{e}^{l}=\left[x_{k+[(l-1) / p]}, x_{k+(l / p)}\right] .
$$

If the element is flagged by the element-wise discontinuity sensor, the discontinuity sensor is further applied to each subcell $I_{e}^{l}$ for $l=1, \ldots, p$. Note that this subdivision is only used to apply the sensor to a low-order entity, that is, the computational mesh is not refined, and the solution is still defined in the whole element $I_{e}$.

This procedure would, at best, increase the accuracy of the shock location with a detected region of width $h / p$ rather than one of size $h$. At worst, if all subcells flag the sensor, the size of the detected area will still cover the element. Notice that increasing $p$ improves the accuracy of this adaptive procedure, while permitting the use of coarser meshes.

\subsection{Subcell viscosity}

Following this idea, the artificial diffusion could be defined within each element as a piecewise function, providing then a closer approximation to the ideal point-wise viscosity.

Here, the simplest approach is considered, which for the examples studied here seems appropriate. A piecewise constant viscosity within each subcell is computed by means of (13) and (15). However, other approaches may be considered. For instance, a Gaussian function centered at the detected region with deviation equal to the total length of the detected subcells is suggested in [15] for element-wise viscosity.

\section{NUMERICAL EXAMPLES}

To demonstrate the capabilities of the proposed approach to preserve the accuracy and capture sharp shock profiles, a variety of test problems are shown in this section. It will be compared with slope limiting procedures, demonstrating the superiority of the proposed approach for both linear and nonlinear problems. All tests have been performed with a third-order explicit RK scheme.

\subsection{Linear advection}

Here, two linear tests are proposed. First, transport of a sinus wave shows that the proposed methodology does not affect the optimal order of convergence of high-order DG methods. Second, a more involved test convecting pulses and Gaussian functions is computed. Both examples are linear initial value problems with periodic boundary conditions and can be defined as

$$
\left\{\begin{array}{l}
u_{t}+u_{x}=0, \quad-1 \leqslant x<1, t>0 \\
u(x, 0)=u_{0}(x)
\end{array}\right.
$$

5.1.1. Transport of a sinus wave. Table I shows error in the $L^{1}$ norm for the initial condition $u_{0}(x)=\sin (\pi x)$ at time $t=2$ for $p=1,2,3,4$ on uniform meshes having $16,32,64,128$, and 256 elements. As expected, the optimal rate of convergence is obtained. Moreover, these errors are at least one order of magnitude smaller compared with those presented in [9] with high-order limiters. Obviously, in this regular linear problem, they are larger than the ones obtained when no shock-capturing procedure is implemented. Table II shows the errors for the DG scheme directly applied to the hyperbolic equation. Note that the accuracy difference between the shock-capturing 
Table I. Transport of a sinus wave: errors in $L^{1}$ norm and rate of convergence.

\begin{tabular}{|c|c|c|c|c|c|c|c|c|}
\hline $\mathrm{N}$ & \multicolumn{2}{|c|}{$p=1$} & \multicolumn{2}{|c|}{$p=2$} & \multicolumn{2}{|c|}{$p=3$} & \multicolumn{2}{|c|}{$p=4$} \\
\hline 16 & $1.64 \mathrm{e}-02$ & & $2.78 \mathrm{e}-04$ & & $4.70 \mathrm{e}-06$ & & $9.10 \mathrm{e}-08$ & \\
\hline 32 & $4.10 \mathrm{e}-03$ & 2.0023 & $3.46 \mathrm{e}-05$ & 3.0036 & $2.92 \mathrm{e}-07$ & 4.0044 & $2.83 e-09$ & 5.0050 \\
\hline 64 & $1.03 \mathrm{e}-03$ & 2.0006 & $4.32 \mathrm{e}-06$ & 3.0009 & $1.83 \mathrm{e}-08$ & 4.0011 & $8.85 \mathrm{e}-11$ & 5.0013 \\
\hline 128 & $2.56 \mathrm{e}-04$ & 2.0001 & $5.40 \mathrm{e}-07$ & 3.0002 & $1.15 \mathrm{e}-09$ & 4.0003 & $2.76 \mathrm{e}-12$ & 5.0003 \\
\hline 256 & $6.40 \mathrm{e}-05$ & 2.0000 & $6.75 \mathrm{e}-08$ & 3.0001 & $7.13 \mathrm{e}-11$ & 4.0001 & $8.63 e-14$ & 5.0004 \\
\hline
\end{tabular}

Table II. Transport of a sinus wave: errors in $L^{1}$ norm without any shock-capturing method.

\begin{tabular}{lcccc}
\hline $\mathrm{N}$ & $p=1$ & $p=2$ & $p=3$ & $p=4$ \\
\hline 16 & $2.47 \mathrm{e}-04$ & $6.20 \mathrm{e}-08$ & $2.06 \mathrm{e}-09$ & $6.37 \mathrm{e}-10$ \\
32 & $1.47 \mathrm{e}-05$ & $1.44 \mathrm{e}-09$ & $1.26 \mathrm{e}-10$ & $3.98 \mathrm{e}-11$ \\
64 & $8.92 \mathrm{e}-07$ & $5.22 \mathrm{e}-11$ & $7.86 \mathrm{e}-12$ & $2.49 \mathrm{e}-12$ \\
128 & $5.48 \mathrm{e}-08$ & $2.68 \mathrm{e}-12$ & $4.91 \mathrm{e}-13$ & $1.55 \mathrm{e}-13$ \\
256 & $3.39 \mathrm{e}-09$ & $1.58 \mathrm{e}-13$ & $3.07 \mathrm{e}-14$ & $9.72 \mathrm{e}-15$ \\
\hline
\end{tabular}

scheme and the standard DG one decreases as the order of the method is increased. Thus, high-order approximations with large elements present reasonable accuracy in smooth problems.

5.1.2. Transport of a combination of Gaussians and pulses. The same linear advection problem is solved with the initial condition

$$
u_{0}(x)= \begin{cases}\frac{1}{6}(G(x, \beta, z-\delta)+G(x, \beta, z+\delta)+4 G(x, \beta, z)) & \text { if } x \in[-0.8,-0.6], \\ 1 & \text { if } x \in[-0.4,-0.2], \\ 1-|10(x-0.1)| & \text { if } x \in[0,0.2], \\ \frac{1}{6}(F(x, \alpha, a-\delta)+G(x, \alpha, a+\delta)+4 G(x, \alpha, a)) & \text { if } x \in[0.4,0.6], \\ 0 & \text { otherwise }\end{cases}
$$

where $G(x, \beta, z)=\exp \left(-\beta(x-z)^{2}\right), F(x, \alpha, a)=\sqrt{\max \left(1-\alpha^{2}(x-\alpha)^{2}, 0\right)}, a=0.5, z=-0.7$, $\delta=0.005, \alpha=10$, and $\beta=\log 2 /\left(36 \delta^{2}\right)$

The solution contains a combination of smooth but narrow Gaussian, a square pulse, a sharp triangle, and a combination of half-ellipses. Moreover, to further accentuate the dissipation introduced by the slope limiter or the artificial diffusion techniques, the solution is computed after a long time, namely $T=8$.

Figure 1 compares the artificial diffusion technique proposed here with the high-order limiter proposed in [9] (described as moments in the figures). Comparisons between these two techniques are always performed with the same number of DOF. In Figure 1(a), 400 DOF are employed; a mesh of 200 elements with $p=1$ and one of 50 elements with $p=7$ are used to compare these techniques. Figure 1(b) corresponds to a mesh of 200 elements with $p=2$ and one of 50 elements and $p=11$.

In general, the artificial diffusion techniques outperform the high-order limiting scheme. This is obvious for high-order elements, which is the natural approach in DG methods. Large high-order elements with the proposed artificial diffusion technique resolve accurately the solution extrema. In contrast, high-order limiting requires, as expected, smaller and low-order elements to capture the features of the transported functions. In other words, it requires $h$-refinement.

Overshoots near discontinuities in $u$ for the square pulse are observed for the artificial diffusion technique. Low-order polynomial approximations present clear localized oscillations in the pulse, see Figure 1. These overshoots should be expected in artificial diffusion methods if no unnecessary added diffusion, which induce over-diffusive solutions, is introduced. Moreover, as noted in [15], the discontinuous nature of the introduced viscosity also favors these overshoots. Nevertheless, it is important to observe that these overshoots are almost negligible for high-order approximations.

Because the amount of artificial diffusion is directly proportional to the limited coefficients, in principle, it seems that there is no need for any discontinuity sensor. If no limiting is necessary, the 

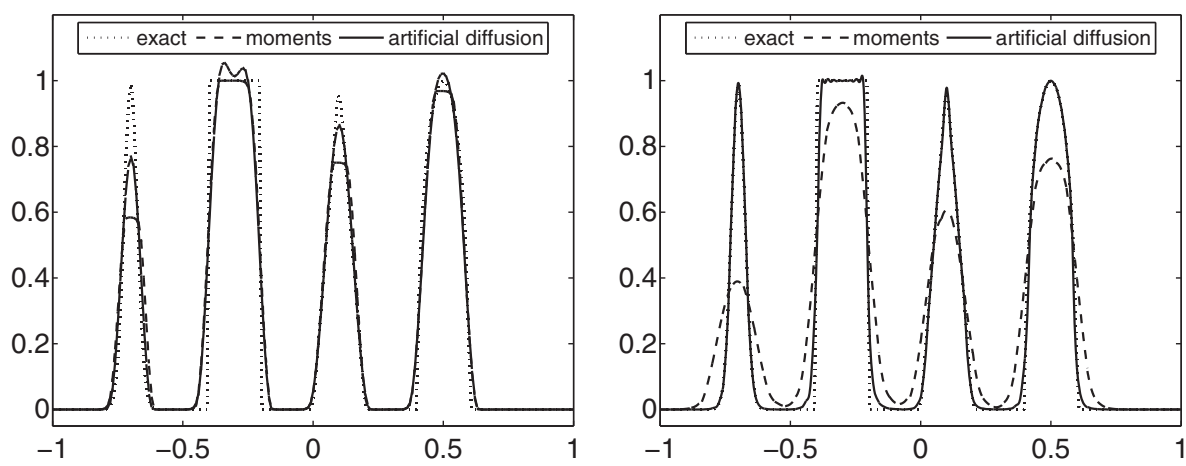

(a)
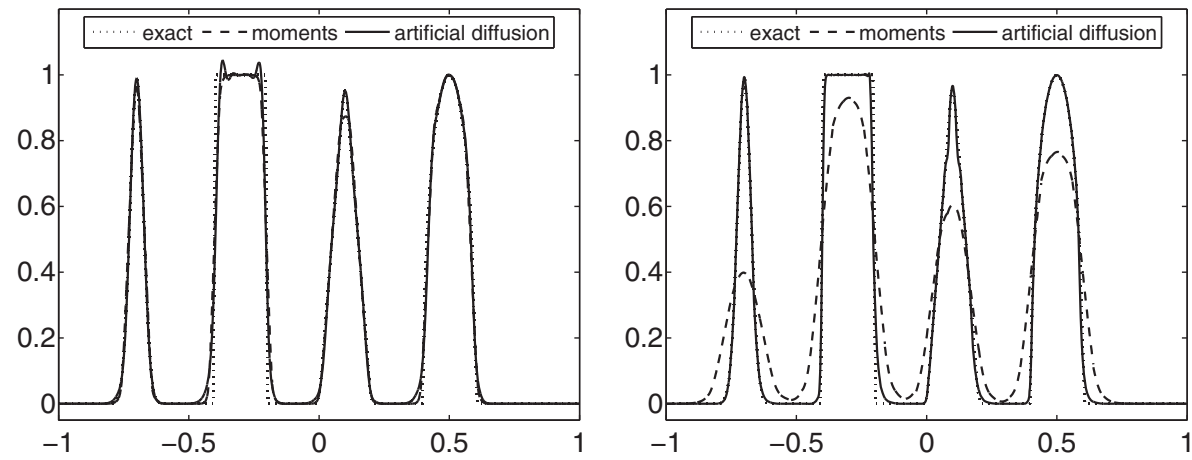

(b)

Figure 1. Combination of Gaussians and pulses: comparison between high-order slope limiter (moments) and artificial diffusion: (a) 400 DOF: 200 elements with $p=1$ (left) and 50 elements with $p=7$ (right) and (b) 600 DOF: 200 elements with $p=2$ (left) and 50 elements with $p=11$ (right).

artificial diffusion is zero. Thus, in a sense, the proposed approach already incorporates a discontinuity sensor. Figure 2 compares the artificial diffusion scheme with and without the discontinuity sensor for 200 and 50 elements. It is clear that such an approach is over-diffusive. Two key aspects contribute to this over-diffusion: constant element-by-element artificial diffusion and the proposed algorithm that always takes the maximum computed diffusion. Note, as previously shown, that using the discontinuity sensor allows both to preclude an over-diffusive method and to reduce the computation overhead only to the elements detected.
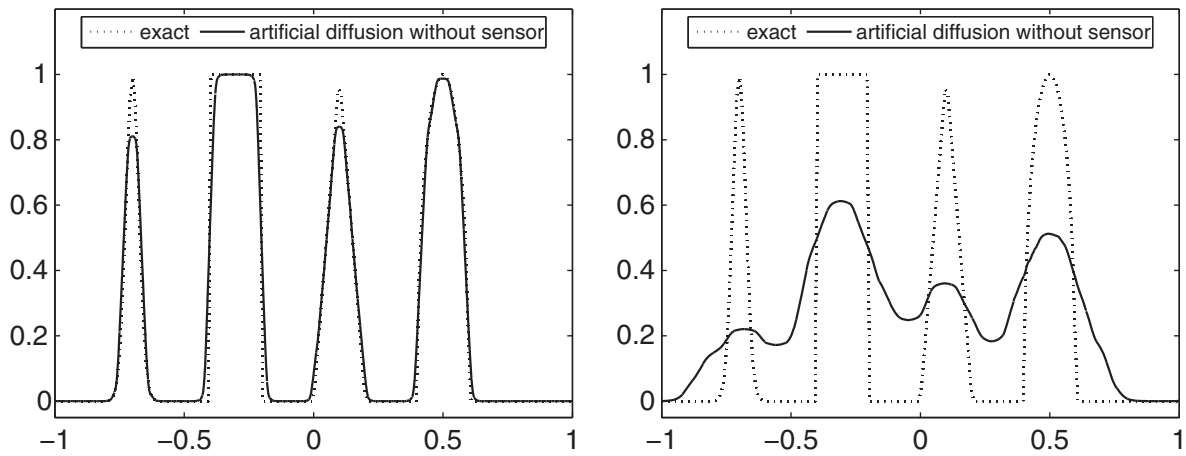

Figure 2. Combination of Gaussians and pulses: no discontinuity sensor for 200 elements with $p=2$ (left) and 50 elements with $p=7$ (right) 


\subsection{Evolution to a steady convection-diffusion problem}

Next, a linear convection-diffusion example is studied,

$$
\left\{\begin{array}{l}
\left.u_{t}+u_{x}-v u_{x x}=1 \quad \text { in }\right] 0,1[, t>0, \\
u(0, t)=u(1, t)=0, \\
u(x, 0)=0 .
\end{array}\right.
$$

For high Peclet numbers, the solution of this problem develops a sharp boundary layer at the rightend of the domain, and the steady solution is reached in a short period of time. Results are presented at $t=1.2$ when the boundary layer is clearly developed. All computations are performed with $v=0.001$, and discretizations are chosen such that Peclet is equal to 10 . Here, the Peclet number is defined as $h / 2 p v$. Note that the characteristic length is the characteristic nodal distance, which in this case is the exact nodal distance because equally spaced nodal elements are employed.

As in the previous example, numerical tests show that the artificial diffusion method outperforms the high-order limiting technique, and in particular, it improves accuracy when coarse meshes and high-order approximations are used. It also ensures smaller inter-element jumps. Polynomial approximations up to $p=11$ are considered, and the number of elements is chosen such that the number of DOF is kept constant (or almost constant). Figure 3 compares results for a discretization with 17 cubic elements and one with 5 elements of degree $p=11$. Note, for instance, that limiting techniques do not to capture the boundary layer.

Moreover, to show that the proposed viscosity is sufficient to capture small boundary layers and non-oscillatory solutions, two artificial viscosity approaches are compared. The artificial diffusion method proposed here, denoted by $\varepsilon$, is compared with a technique that adds constant artificial viscosity of order $h / p$, denoted by $\varepsilon^{h / p}$, as suggested in [14]. Results for the previous discretizations
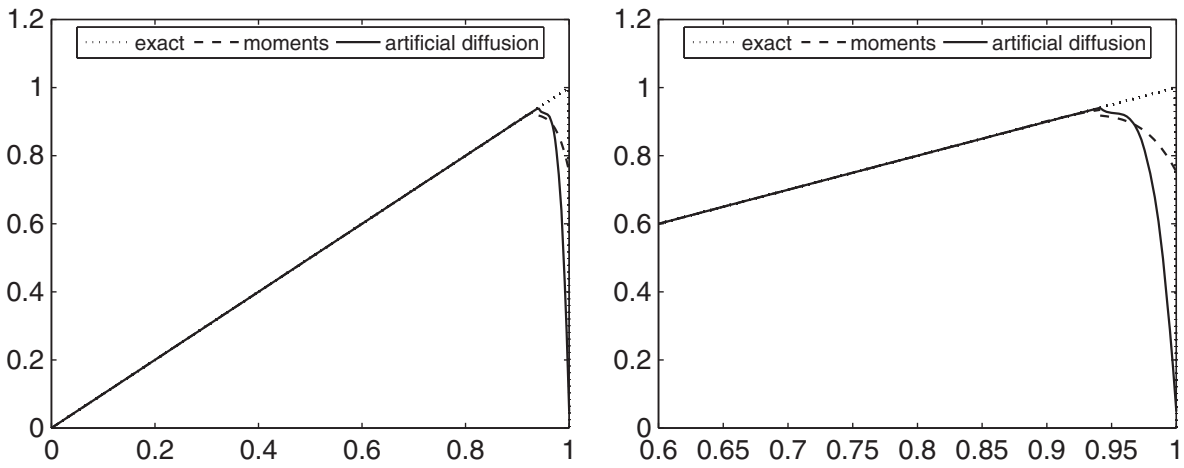

(a)
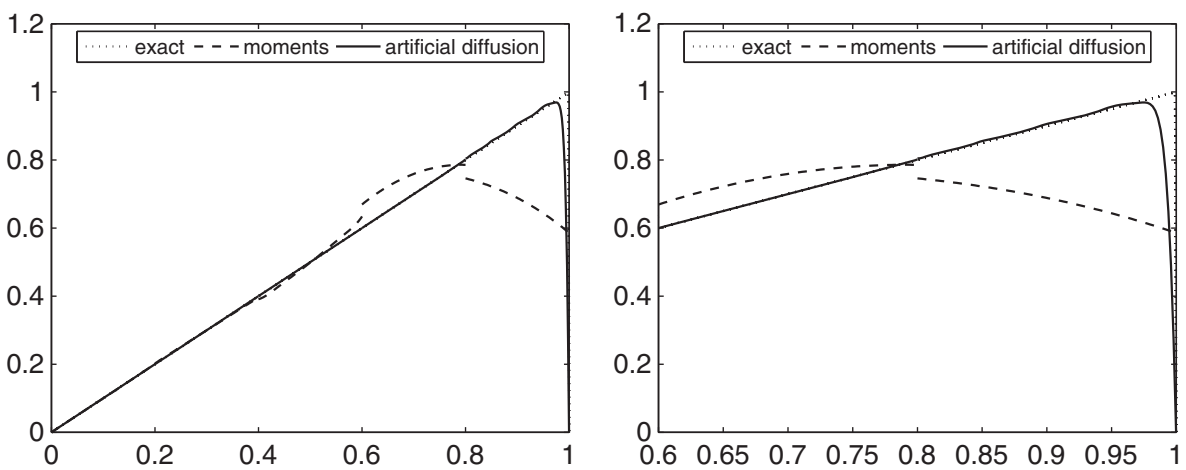

(b)

Figure 3. Evolution to a steady convection-diffusion problem: figures on the right show the detail around the boundary layer: (a) 17 elements and $p=3$ and (b) 5 elements and $p=11$. 


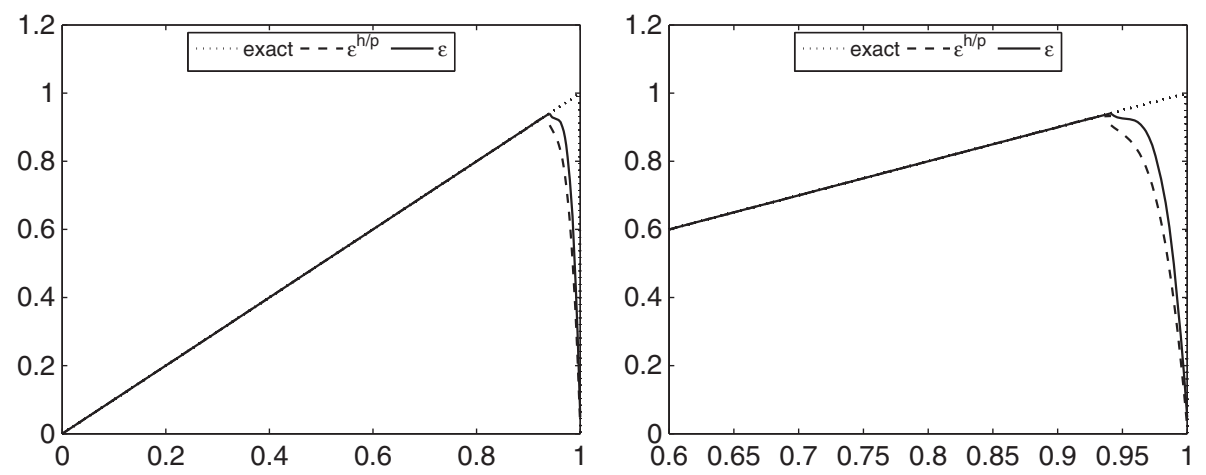

(a)
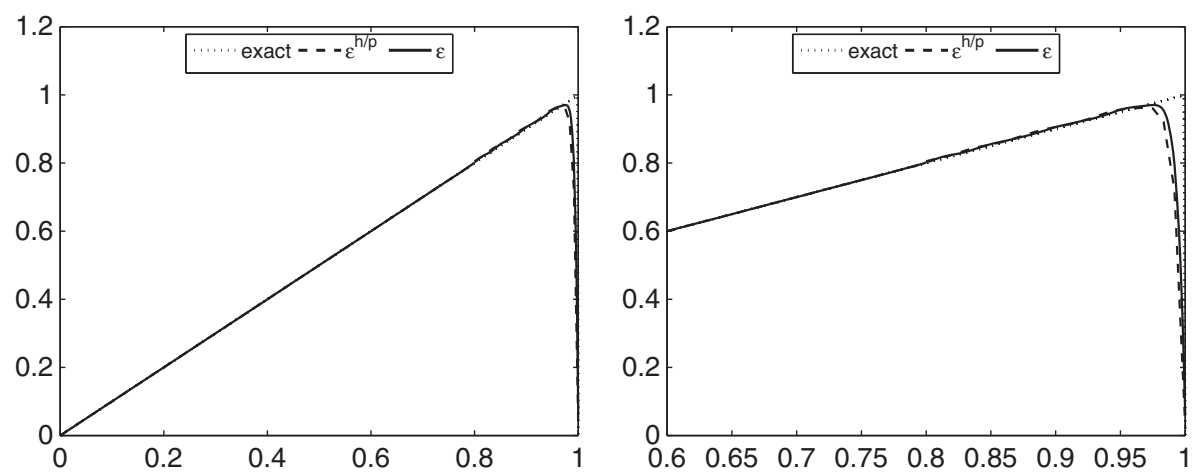

(b)

Figure 4. Evolution to a steady convection-diffusion problem: comparison between a diffusion of order $h / p$ and the proposed technique: (a) 17 elements and $p=3$ and (b) 5 elements and $p=11$.
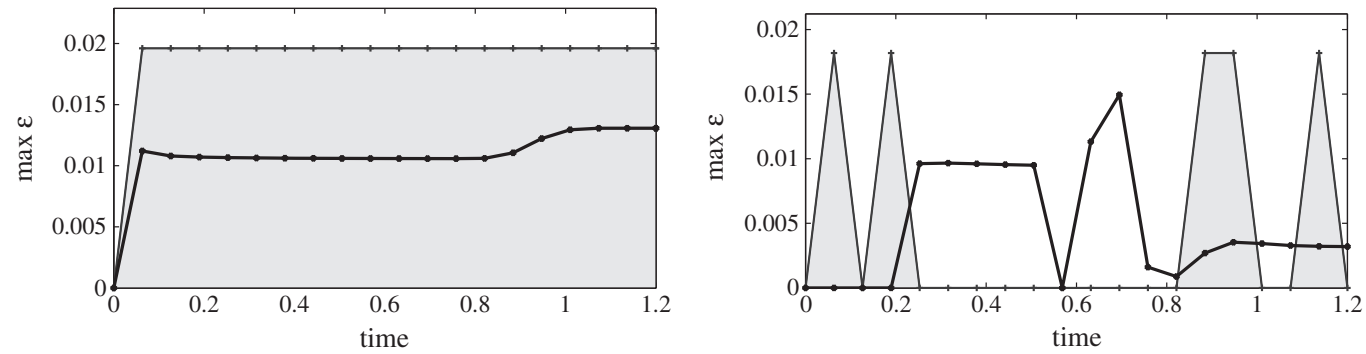

Figure 5. Evolution to a steady convection-diffusion problem: evolution of artificial diffusion with time; the order $h / p$ viscosity is filled in gray, and the proposed diffusion as a solid black line; (left) $p=3$ and (right) $p=11$.

are shown in Figure 4, for $p=3$ on the top plots and $p=11$ on the bottom ones. Qualitative similar solution profiles are obtained with both techniques. However, numerical evidence indicates that the added viscosity for the approach proposed here is smaller, for any degree of approximation, than $h / p$. In fact, Figure 5 shows the amount of diffusion introduced in the last element at each time step for both cases.

To further differentiate both artificial diffusion techniques, the $L^{\infty}$ and $L^{1}$ error norms are computed in the region containing the boundary layer, that is, for $0.8 \leqslant x \leqslant 1$. Results are shown in Table III. The $L^{1}$ error is also computed for the high-order limiter. The $L^{\infty}$ error is not shown for the slope limiting technique because it is too large in the vicinity of $x=1$. Note that the proposed methodology always presents the smallest errors. 
Table III. Evolution to a steady convection-diffusion problem: $L^{1}$ and $L^{\infty}$ error norms for $x \in[0.8,1]$.

\begin{tabular}{|c|c|c|c|c|c|c|c|c|}
\hline & \multicolumn{4}{|c|}{$L^{1}$ error } & \multicolumn{4}{|c|}{$L^{\infty}$ error } \\
\hline & $p=3$ & $p=5$ & $p=8$ & $p=11$ & $p=3$ & $p=5$ & $p=8$ & $p=11$ \\
\hline Limiter & 0.0061 & 0.0154 & 0.0334 & 0.0438 & & & & \\
\hline$\varepsilon=h / p$ & 0.0184 & 0.0111 & 0.0086 & 0.0062 & 0.8115 & 0.7721 & 0.7214 & 0.7493 \\
\hline$\varepsilon$ proposed & 0.0133 & 0.0080 & 0.0070 & 0.0047 & 0.7437 & 0.7719 & 0.7001 & 0.6487 \\
\hline
\end{tabular}
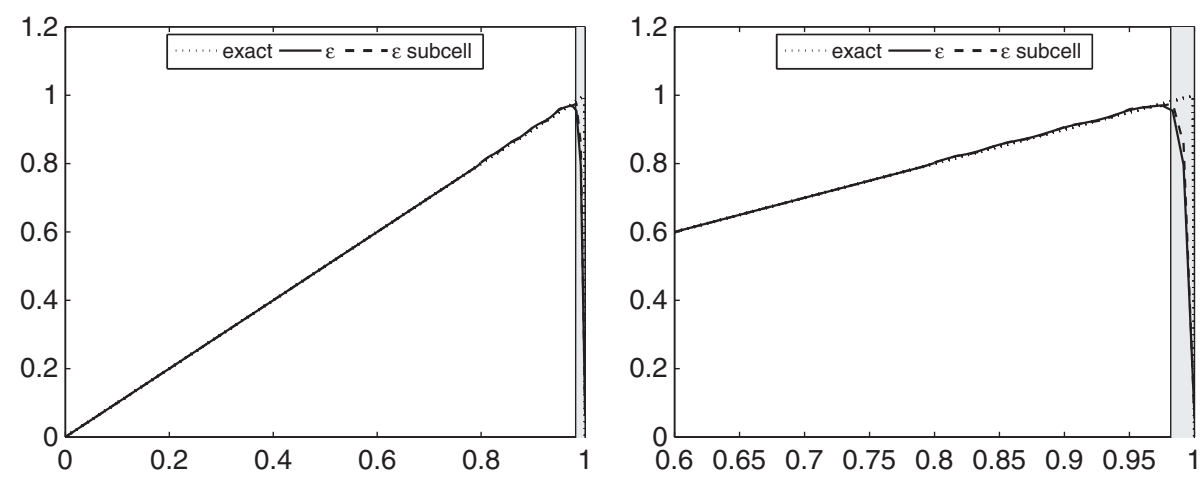

Figure 6. Evolution to a steady convection-diffusion problem: comparison between the constant elementby-element artificial diffusion approach and the subcell one. The region filled in gray is the subcell where the artificial diffusion is nonzero.

Finally, Figure 6 shows the approximation of degree $p=11$ over the mesh of 5 elements with the subcell artificial diffusion technique. A zoom on two elements is also shown. The constant elementby-element artificial diffusion approach imposes a finite viscosity on $[0.8,1]$. But with the subcell approach, the artificial diffusion is only added in a region ten times smaller, that is, $h /(p-1)$.

\subsection{Burgers' equation}

To generalize the previous conclusions also for nonlinear problems, the inviscid Burgers' equation with periodic boundary conditions is solved

$$
\left\{\begin{array}{l}
u_{t}+f_{x}(u)=0 \\
u(x, 0)=\frac{1}{2}+\sin (2 \pi x) .
\end{array} \text { in }\right] 0,1[, t>0
$$

The solution of this problem forms a shock at $t=0.25$ that moves to the right. Solutions are compared at $t=0.5$.

Figure 7 compares a high-order slope limiting procedure and the proposed artificial diffusion technique. The problem is solved on a 10-element mesh with $p=5$ and $p=10$. Note that the high-order limiter proposed in [8] gives, in this case, the same results for $p=5$ and $p=10$. Highorder limiting starts with the highest order coefficient and stops limiting when the solution is nearly TVD. In this example, the adaptive limiter limits coefficients for both $p=5$ and $p=10$ to the same order, which is $\ll 5$, and consequently, same results are obtained. On the contrary, for the artificial diffusion method, accuracy improves as the degree is increased as well as the shock sharpness, see Figure 8 .

The proposed approach is also compared with a constant artificial viscosity of order $h / p$, denoted by $\varepsilon^{h / p}$, as suggested in [14]. Figure 9 shows the obtained solution for $p=3,5,8$, and 11 on a 10-element mesh. Both techniques produce similar shock profiles as the order of the approximation is increased. Note, however, that the proposed viscosity is smaller than the one of order $h / p$. 


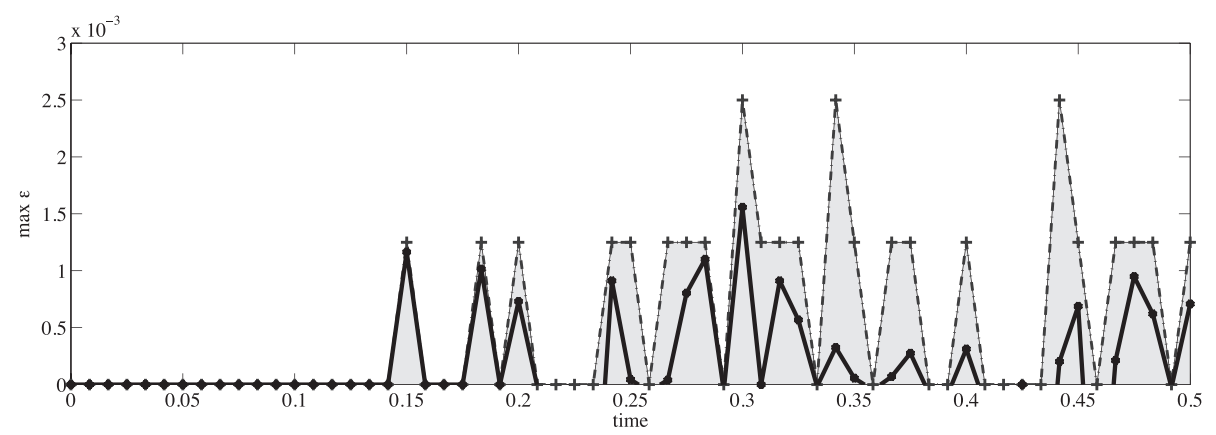

Figure 10. Burgers' equation: evolution of the added viscosity for $p=8$. Artificial diffusion of order $h / p$ (gray) and proposed diffusion (solid black line)

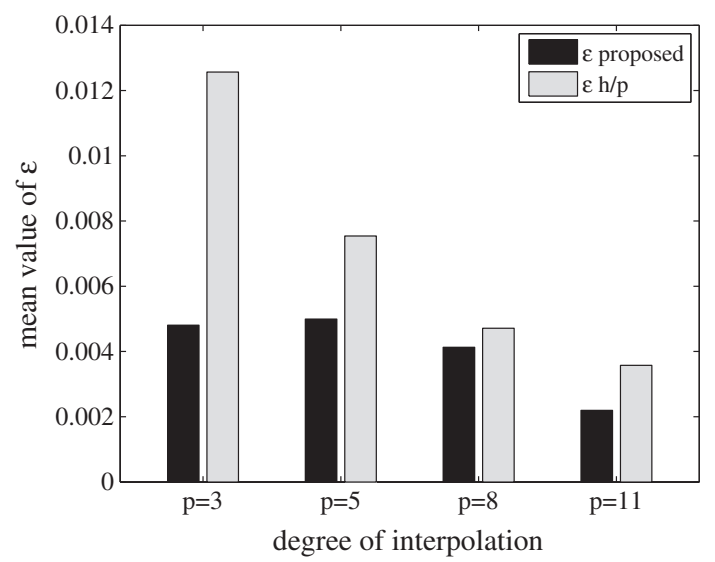

Figure 11. Burgers' equation: mean added viscosity during the computation for different degrees of approximation and both artificial diffusion techniques.

Note that, in general, less added viscosity leads to less error. To better compare both approaches as well as their accuracy, the total amount of introduced viscosity is computed. In contrast to the previous example here, the shock evolves in time, and hence, it may change from one element to another along the computation. Hence, to make a fair comparison, the total viscosity footprint is computed as

$$
\tilde{\varepsilon}(t)=\sum_{e=1}^{\mathrm{n}_{\mathrm{el}}} \int_{\Omega_{e}} \varepsilon(x, t) d x \quad \text { for } t \in[0, T] .
$$

Figure 10 shows the evolution of the added diffusion for a discretization of degree $p=8$ and a mesh of 10 elements for both methods. The viscosity proposed here is always smaller than the one of order $h / p$. Similar results are obtained for different discretizations. The mean value of the added viscosity along all the process is also computed. That is,

$$
\bar{\varepsilon}=\frac{1}{T} \int_{0}^{T} \tilde{\varepsilon}(t)
$$

Figure 11 compares both artificial diffusion approaches for different degrees of approximation. Note that the amount of viscosity required decreases as the degree of approximation increases.

Finally, the subcell artificial diffusion is also applied to the same 10-element mesh with $p=8$, see Figure 12. Note that the vertical dashed grid lines indicate the elements. On the left, at $t=0.25$, the shock lies inside one element. Whereas on the right, at $t=0.5$, the shock spans between two 

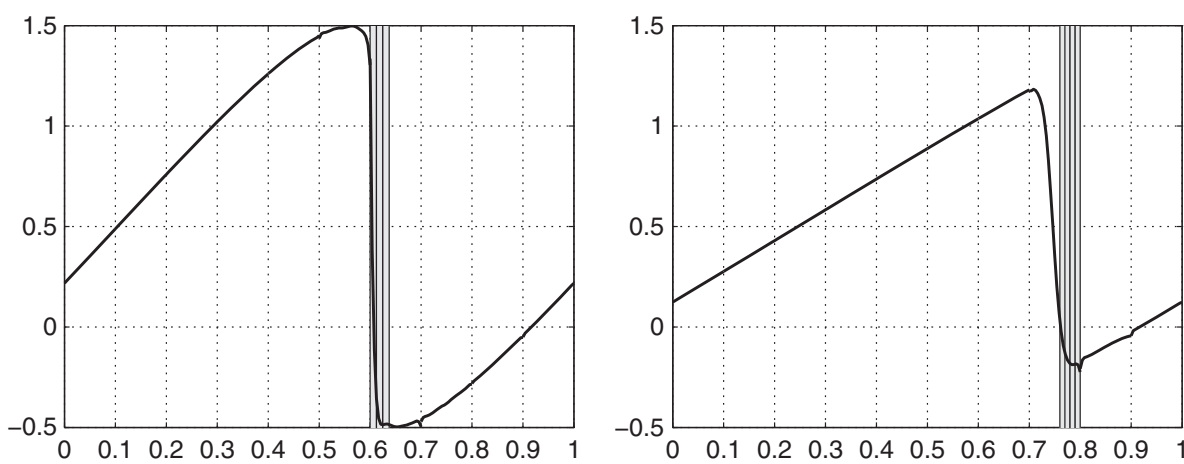

Figure 12. Burgers' equation: solution at time $t=0.25$ (left) and $t=0.5$ (right). The footprint diffusion is added and is filled in gray.

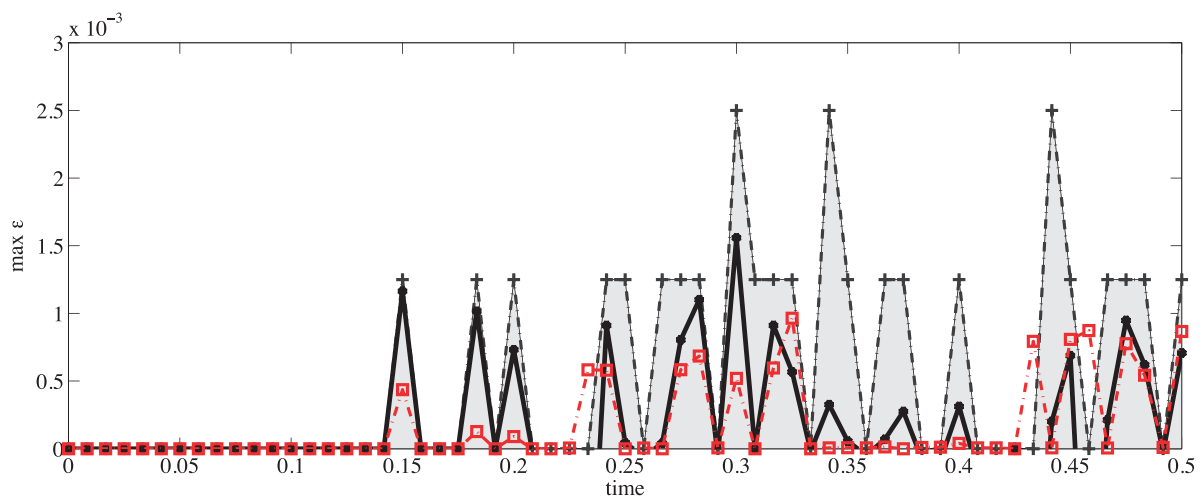

Figure 13. Burgers' equation: evolution of the viscosity for $p=8$. Artificial diffusion of order $h / p$ (gray), element-wise diffusion (continuous line), and subcell diffusion (dashed line).

elements. In both cases, the area where viscosity is added is smaller than an element. Moreover, for $t=0.5$, instead of spreading the artificial diffusion over two elements, it is reduced to a small region around their boundary.

The amount of added viscosity with the subcell detection is determined using Equation 17. Figure 13 compares this approach with the previous ones and clearly shows that the subcell approach reduces the total amount of added viscosity.

In summary, large high-order elements do not imply a more of artificial viscosity, and the subcell approach induces qualitatively similar shock profiles and a smaller viscosity footprint.

\subsection{Euler equations}

Finally, to show the applicability of this approach to systems of nonlinear equations, the onedimensional Euler equations are solved for the classical Sod's shock tube problem, see [27]. That is, the one-dimensional Euler equations $\boldsymbol{U}_{t}+\boldsymbol{F}(\boldsymbol{U})_{x}=\mathbf{0}$ with

$$
\boldsymbol{U}=(\rho, \rho v, \rho E)^{T} \text { and } \boldsymbol{F}(\boldsymbol{U})=\left(\rho v, \rho v^{2}+p,(\rho E+p) v\right)^{T}
$$

are solved subject to the initial conditions

$$
(\rho, v, p)= \begin{cases}(3,0,3) & \text { if } 0 \leqslant x \leqslant 0.5 \\ (1,0,1) & \text { if } 0.5<x \leqslant 1 .\end{cases}
$$

with $\gamma=1.4$.

The artificial diffusion approach consist in adding a nonconstant artificial viscosity tensor to the previous system of conserved quantities. In fact, the proposed artificial diffusion approach is applied 
independently to the three uncoupled equations defined by the characteristic variables (also called Riemann variables), see [28]. Recall that the one-dimensional Euler equations diagonalize with the matrix of the right eigenvectors of the Jacobian of the flux $\boldsymbol{F}(\boldsymbol{U})$. Thus, an artificial viscosity is obtained for each equation. This diagonal tensor of added viscosities is mapped back into the conserved variables by multiplication with the matrix of right eigenvectors, obtaining a nonconstant artificial viscosity tensor for the system of conserved quantities.

Solution profiles at time $t=0.2$ for density, velocity, and pressure for the Sod's shock tube problem are reported in Figure 14. Note that entropy is the unique variable used in the discontinuity sensor. These results are compared with high-order limiting techniques. Figure 14 demonstrates the
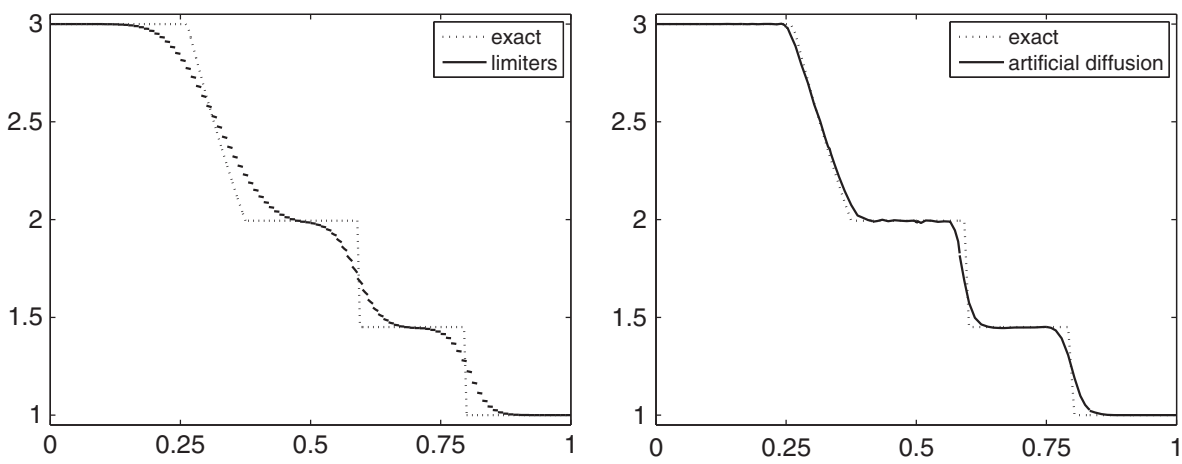

(a)
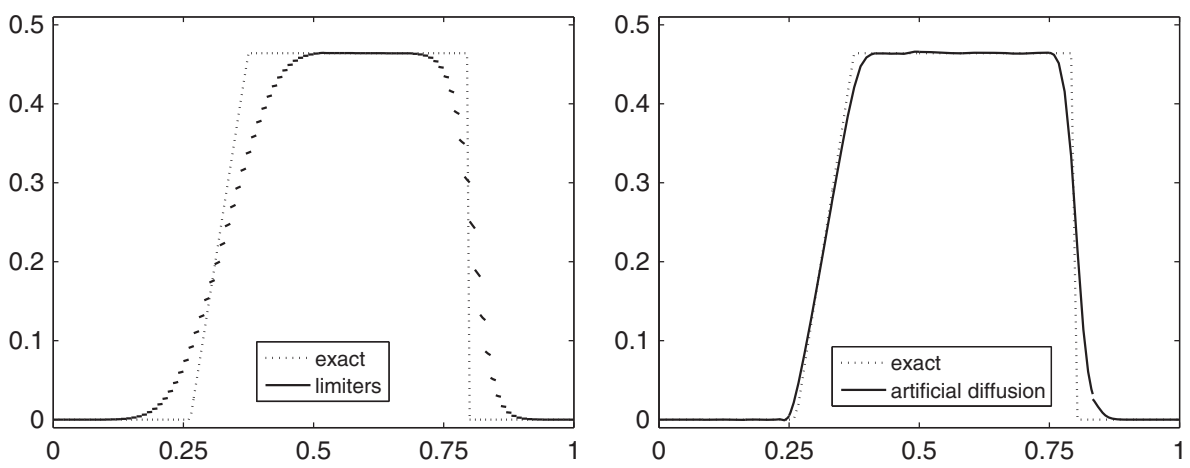

(b)
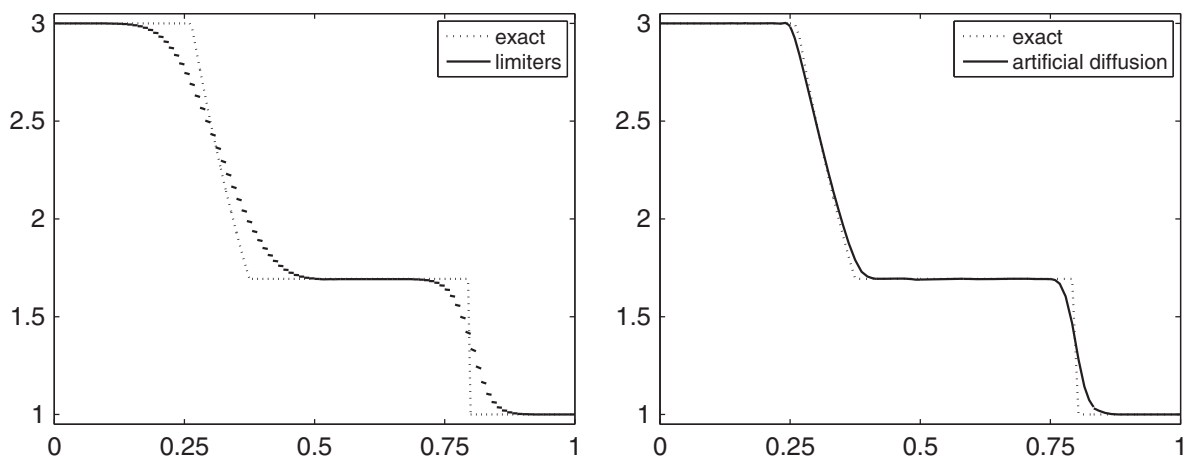

(c)

Figure 14. Euler equations: comparison between high-order limiters with 300 DOF (left) and artificial diffusion method with 132 DOF (right). (a) density (b) velocity and (c) pressure. 
Table IV. Euler equations: $L^{1}$ errors within each variable.

\begin{tabular}{lcc}
\hline & Limiters & Artificial diffusion \\
\hline$\rho$ & $1.08 \mathrm{e}-02$ & $2.33 \mathrm{e}-03$ \\
$v$ & $8.30 \mathrm{e}-03$ & $2.32 \mathrm{e}-04$ \\
$p$ & $2.75 \mathrm{e}-02$ & $1.00 \mathrm{e}-03$ \\
\hline
\end{tabular}

superiority of the proposed approach. On the left, the limiter solution is plotted, and it is obtained with 300 DOF (100 elements with $p=2$ ). On the right, the results of the proposed approach are depicted; they correspond to a discretization with 132 DOF (12 elements with $p=10)$. The artificial diffusion approach clearly shows sharper fronts with less than half the number of DOF. In this transient problem, shocks, which in some cases are smaller than one element size, are captured accurately with the artificial viscosity. Note also that no oscillations are noticeable.

Finally, Table IV shows the $L^{1}$ error for both methods (high-order limiter and proposed approach). The error is evaluated for each physical quantity, and as expected, the errors induced by the proposed approach are one order smaller than the ones obtained with a high-order limiter.

\section{CONCLUDING REMARKS}

A novel artificial diffusion methodology for high-order discontinuous Galerkin methods is proposed. In fact, the extensive experience in limiting techniques is used to define how much viscosity must be introduced in this artificial diffusion method. That is, the equivalent viscosity introduced by a high-order limiting technique is determined and then explicitly introduced in the equations. This approach precludes reducing systematically to first-order the solution in the vicinity of the shock. This is crucial because no mesh refinement is systematically required. Moreover, this approach consistently introduces a diffusion smaller than $\mathcal{O}(h / p)$, which, in general, also implies a more accurate solution. A simple extension of the proposed approach further locates the shock within the element in subcells smaller than the element size. Although explicit time integration is used here, artificial viscosity can also be implemented in the framework of implicit integrators. Numerical examples range from linear to nonlinear problems, as well as systems of equations. They all show that sharp shock profiles, thinner than the element size, are obtained without the need of any refinement procedures. Moreover, they demonstrate the superiority of the proposed method in front of classical limiting techniques and also its efficiency, in particular, when the polynomial degree is increased.

\section{ACKNOWLEDGEMENTS}

The authors would like to thank Generalitat de Catalunya AGAUR (2009SGR875) for the financial support.

\section{REFERENCES}

1. Cockburn B, Shu CW. TVB Runge-Kutta local projection discontinuous Galerkin finite element method for conservation laws. II. General framework. Mathematics of Computation 1989; 52(186):411-435.

2. Cockburn B, Lin SY, Shu CW. TVB Runge-Kutta local projection discontinuous Galerkin finite element method for conservation laws. III. One-dimensional systems. Journal of Computational Physics 1989; 84(1):90-113.

3. Shu CW. Total-variation-diminishing time discretizations. Society for Industrial and Applied Mathematics. Journal on Scientific and Statistical Computing 1988; 9(6):1073-1084.

4. Shu CW, Osher S. Efficient implementation of essentially nonoscillatory shock-capturing schemes. Journal of Computational Physics 1988; 77(2):439-471.

5. Gottlieb S, Shu CW, Tadmor E. Strong stability-preserving high-order time discretization methods. SIAM Review 2001; 43(1):89-112.

6. LeVeque RJ. Numerical Methods for Conservation Laws (2nd edn), Lectures in Mathematics ETH Zürich. Birkhäuser Verlag: Basel, 1992.

7. Burbeau A, Sagaut P, Bruneau CH. A problem-independent limiter for high-order Runge-Kutta discontinuous Galerkin methods. Journal of Computational Physics 2001; 169(1):111-150.

8. Biswas R, Devine KD, Flaherty JE. Parallel, adaptive finite element methods for conservation laws. Applied Numerical Mathematics 1994; 14(1-3):255-283. 
9. Krivodonova L. Limiters for high-order discontinuous Galerkin methods. Journal of Computational Physics 2007; 226(1):879-896.

10. Qiu J, Shu CW. Runge-Kutta discontinuous Galerkin method using WENO limiters. SIAM Journal on Scientific Computing 2005; 26(3):907-929.

11. Huerta A, Casoni E, Peraire J. A simple shock-capturing technique for high-order discontinuous Galerkin methods. International Journal for Numerical Methods in Fluids 2011. Available from: http:/dx.doi.org/10.1002/fld.2654.

12. von Neumann J, Richtmyer RD. A method for the numerical calculation of hydrodynamic shocks. Journal of Applied Physics 1950; 21:232-237.

13. Cockburn B. Devising discontinuous Galerkin methods for non-linear hyperbolic conservation laws. Journal of Computational and Applied Mathematics 2001; 128(1-2):187-204.

14. Persson P-O, Peraire J. Sub-cell shock capturing for discontinuous Galerkin methods. In Collection of Technical Papers - 44th AIAA Aerospace Sciences Meeting, Vol. 2, 2006; 1408-1420. AIAA-2006-0112, Reno, Nevada.

15. Barter GE, Darmofal DL. Shock capturing with PDE-based artificial viscosity for DGFEM: part I. formulation. Journal of Computational Physics 2010; 229(5):1810-1827.

16. Casoni E, Peraire J, Huerta A. One-dimensional shock-capturing for high-order discontinuous Galerkin methods. In ECCOMAS Multidisciplinary Jubilee Symposium, Computational Methods in Applied Sciences, Vol. 14, Eberhardsteiner J, Hellmich C, Mang HA, Périaux J (eds). Springer: Netherlands, 2009; 307-325.

17. Arnold DN. An interior penalty finite element method with discontinuous elements. SIAM Journal on Numerical Analysis 1982; 19(4):742-760.

18. Bassi F, Rebay S. A high-order accurate discontinuous finite element method for the numerical solution of the compressible Navier-Stokes equations. Journal of Computational Physics 1997; 131(2):267-279.

19. Bassi F, Rebay S. Numerical evaluation of two discontinuous Galerkin methods for the compressible Navier-Stokes equations. International Journal for Numerical Methods in Engineering 2001; 40(10):197-207.

20. Cockburn B, Shu C-W. Runge-Kutta discontinuous Galerkin methods for convection-dominated problems. Journal of Scientific Computing 2001; 16(3):173-261.

21. Peraire J, Persson P-O. The compact discontinuous Galerkin (CDG) method for elliptic problems. Society for Industrial and Applied Mathematics. Journal on Scientific Computing 1988; 30(4):1806-1824.

22. Cockburn B, Shu C-W. The local discontinuous Galerkin method for time-dependent convection-diffusion systems. SIAM Journal on Numerical Analysis 1998; 35(6):2440-2463.

23. Osher S. Riemann solvers, the entropy condition, and difference approximations. SIAM Journal on Numerical Analysis 1984; 21(2):217-235.

24. Hesthaven JS, Warburton T. Nodal Discontinuous Galerkin Methods, Texts in Applied Mathematics, Vol. 54. Springer: New York, 2008. Algorithms, analysis, and applications.

25. Casoni E, Peraire J, Huerta A. Un método de captura de choques basado en las funciones de forma para galerkin discontinuo en alto orden. Revista Internacional de Métodos Numéricos en Ingeniería 2011. To appear.

26. Folland GB. Fourier Analysis and its Applications, The Wadsworth \& Brooks/Cole Mathematics Series. Wadsworth \& Brooks/Cole Advanced Books \& Software: Pacific Grove, CA, 1992.

27. LeVeque RJ. Finite Volume Methods for Hyperbolic Problems, Cambridge Texts in Applied Mathematics. Cambridge University Press: Cambridge, 2002.

28. Donea J, Huerta A. Finite Element Methods for Flow Problems. John Wiley \& Sons: Chichester, 2003. 